\title{
EFEITO DO PILATES SOBRE A FLEXIBILIDADE DO TRONCO E AS MEDIDAS ULTRASSONOGRÁFICAS DOS MÚSCULOS ABDOMINAIS
}

\author{
EFFECT OF PILATES ON TRUNK FLEXIBILITY AND ULTRASOUND MEASURES \\ OF ABDOMINAL MUSCLES
}

\begin{abstract}
EFECTO DEL PILATES EN LA FLEXIBILIDAD DEL TORSO Y LAS MEDIDAS DE LOS MÚSCULOS ABDOMINALES POR ULTRASONIDO
\end{abstract}

Gisela Rocha de Siqueira (Fisioterapeuta)

Geisa Guimarães de Alencar ${ }^{1}$ (Fisioterapeuta)

Érika da Cruz de Melo Oliveira (Fisioterapeuta)

Vanessa Queiroz Melo Teixeira' (Fisioterapeuta)

1. Universidade Federal de Pernambuco, Recife, PE, Brasil.

\section{Correspondência:}

Rua Capitão Rui Lucena, 160. Apto 904 Boa Vista. Recife, PE, Brasil. 50070-080. geisa.guimaraes@hotmail.com

\begin{abstract}
RESUMO
Introdução: a Pilates consiste em um método de treinamento físico e mental que trabalha a flexibilidade e a força muscular. Esse método prioriza fortalecer o conjunto de músculos responsável pelo controle de tronco, chamado de Powerhouse e, consequentemente, aumentar o trofismo dos músculos abdominais (reto do abdome, oblíquo interno e externo, transverso do abdome), glúteos, músculos do períneo e paravertebrais lombares. Objetivo: avaliar o efeito do método Pilates sobre o trofismo do grupamento abdominal e na flexibilidade do tronco, comparado à aplicação de uma técnica tradicional de fortalecimento dos músculos abdominais e alongamentos estáticos em mulheres saudáveis. Métodos: treze mulheres sedentárias e saudáveis na faixa etária entre 18 e 25 anos foram submetidas a 10 sessões de Pilates (grupo Pilates, $n=6$ ) e 10 sessões de alongamento e fortalecimento tradicionais (grupo controle, $n=7$ ). Antes e após as intervenções, o trofismo da musculatura abdominal das voluntárias foi avaliado através do ultrassom e a flexibilidade do tronco através do flexímetro. Resultados: no grupo Pilates, após a intervenção, houve um aumento significativo das medidas ultrassonográficas do reto do abdome, da amplitude da rotação para direita e esquerda e da inclinação da coluna vertebral para a esquerda. No grupo controle, observou-se melhora apenas na rotação para a esquerda. Conclusão: o número de sessões pode ter sido insuficiente para que ocorresse aumento do trofismo de todos os músculos avaliados e da flexibilidade do tronco. É importante investir em pesquisas sobre Pilates, especialmente com a utilização de métodos de imagem mais acurados.
\end{abstract}

Palavras-chave: fisioterapia, ultrassom, abdome, técnicas de exercício e de movimento.

\begin{abstract}
Introduction: Pilates is a method of physical and mental training that works on flexibility and muscle strength. This method prioritizes strengthening the set of muscles responsible for trunk control, called Powerhouse, and thereby increase the trophysm of the abdominal muscles (rectus abdominis, internal and external oblique, transversus abdominis), gluteus, perineal and lumbar paraspinal muscles. Objective: to evaluate the effect of the Pilates method on the trophysm of abdominal and trunk flexibility compared to the application of a traditional technique to strengthen the abdominal muscles and static stretching in healthy women. Methods: thirteen healthy sedentary women, aged between 18 and 25 years underwent 10 sessions of Pilates (Pilates group, $n=6$ ) and 10 sessions of traditional stretching and strengthening (control group, $n=7$ ). Before and after the intervention, the trophysm of the abdominal muscles of the volunteers was assessed using ultrasound, and trunk flexibility was evaluated through the fleximeter. Results: after the intervention, there was a significant increase in ultrasound measures of the rectus abdominis muscle, the range of the rotation to right and left, and tilt of the spine to the left in the Pilates group. In the control group, improvement was observed only in the rotation to the left. Conclusion: the number of sessions may have been insufficient for the increase in the trophysm of all muscles tested and the flexibility of the trunk to occur. It is important to invest in research on Pilates, especially with the use of more accurate imaging methods.
\end{abstract}

Keywords: physical therapy, ultrasonics, abdomen, exercise movement techniques.

\section{RESUMEN}

Introducción: Pilates es un método de entrenamiento físico y mental que trabaja en la flexibilidad y la fuerza muscular. Este método da prioridad a fortalecer el grupo de músculos responsables por el control del torso, llamado de Powerhouse, y con ello aumentar el tropismo de los músculos abdominales (recto abdominal, oblicuo interno y externo, transverso del abdomen), los glúteos, los músculos del perineo y paravertebrales lumbares. Objetivo: evaluar el efecto del método Pilates en el tropismo de agrupación abdominal y flexibilidad del torso en comparación con la aplicación de una técnica tradicional para fortalecer los músculos abdominales y estiramiento estático en mujeres sanas. Métodos: 13 mujeres sedentarias sanas, con edades comprendidas entre 18 y 25 años fueron sometidas a 10 sesiones de Pilates (grupo Pilates, $n=6$ ) y 10 sesiones de estiramiento y fortalecimiento tradicionales (grupo control, 
$n=7$ ). Antes y después de la intervención, el tropismo de los músculos abdominales de las voluntarias se evaluó mediante ultrasonido y la flexibilidad del tronco a través del flexímetro. Resultados: en el grupo Pilates, después de la intervención, hubo un aumento significativo en las medidas de ultrasonido del recto del abdomen, la magnitud de la rotación a la derecha y a la izquierda y la inclinación de la columna vertebral hacia la izquierda. En el grupo control, la mejora sólo se observó en la rotación a la izquierda. Conclusión: el número de sesiones puede haber sido insuficiente para producir mayor tropismo de todos los músculos evaluados y flexibilidad del torso. Es importante invertir en la investigación de Pilates, especialmente con el uso de métodos de imagen más precisos.

Palabras clave: fisioterapia, ultrasonido, abdomen, técnicas de ejercicio con movimientos.

\section{INTRODUÇÃO}

O Pilates trata-se de uma filosofia de treinamento do corpo e mente que realiza o trabalho muscular em baixa velocidade 1 . Foi criado por Joseph Pilates em 1918 e tem como objetivo conseguir um controle preciso do corpo ${ }^{2}$ através de uma variedade de exercícios executados em solo ou em aparelhos próprios'.

Indicado para qualquer faixa etária, este método contém as modificações e adaptações adequadas para os diferentes indivíduos e patologias, respeitando as características e limitações de cada pessoa. O método engloba exercícios nos quais são utilizados seis princípios: concentração, controle, precisão, fluidez do movimento, respiração e contração do centro de força ${ }^{3}$.

A powerhouse (centro de força) constitui-se pelos músculos abdominais (reto abdominal, oblíquo interno e externo, transverso do abdome), glúteos, músculos do períneo e paravertebrais lombares, que são responsáveis pela estabilização estática e dinâmica do cor$\mathrm{po}^{4-6}$ e, segundo o método, estes músculos devem ser contraídos durante a expiração, garantindo a manutenção da postura correta, com menor gasto energético aos movimentos ${ }^{7,8}$ e diminuição do risco de lesões ${ }^{9}$.

Entre os benefícios deste método podem ser destacados principalmente a melhora da flexibilidade, o aumento da força muscular ${ }^{10} \mathrm{e}$, possivelmente, do trofismo (espessura) muscular, uma vez que existe relação positiva entre a força e hipertrofia ${ }^{11}$. Sua prática vem ficando a cada dia mais popular, embora as evidências científicas sobre o método ainda sejam escassas.

Para avaliação do trofismo muscular, alguns métodos podem ser aplicados, tais como o a ressonância magnética, a tomografia computadorizada ou até cálculos de perímetros musculares juntamente com dobras cutâneas ${ }^{12-14}$. No entanto, um exame que se destaca na avaliação do trofismo é o Ultrassom, que é uma técnica de obtenção de imagens em tempo real de baixo custo, além de não ser invasivo e não possuir propriedades ionizantes como os Raios $X^{15}$.

Além do trofismo, a flexibilidade parece ter uma boa resposta em pessoas praticantes de Pilates. O equipamento que se sobressai em pesquisas para a avaliação da flexibilidade é o flexímetro, que a partir de um sistema pendular gravitacional, oferece precisão e praticidade nas mensurações dos movimentos angulares, por permitir ser fixado no corpo, além de oferecer maior confiabilidade nas leituras das medidas, uma vez que a indicação do ângulo é produzida por efeito da gravidade, minimizando os erros de interpretação do eixo longitudinal correspondente ${ }^{16}$.

Diante da expansão do número de adeptos a este método ao longo dos anos, a proposta do presente estudo consiste em avaliar o efeito do método Pilates no trofismo do grupamento abdominal e na flexibilidade do tronco comparado à aplicação de uma técnica tradicional de fortalecimento dos músculos abdominais e alongamentos estáticos em mulheres saudáveis.

\section{MÉTODOS}

Os procedimentos executados foram aprovados pelo Comitê de Ética em Pesquisa (CAAE: 18352013.3.0000.5208) da Instituição. Todos os voluntários assinaram o Termo de Consentimento Livre e Esclarecido (TCLE), de acordo com a Resolução 466/12.

Trata-se de um estudo de intervenção, randomizado e realizado com mulheres jovens, eutróficas, sedentárias e saudáveis.

A amostra foi composta por mulheres entre 18 e 25 anos, foram excluídas àquelas com o IMC fora dos limites de normalidade (maior que $24,9 \mathrm{~kg} / \mathrm{m}^{2}$ e menor que $\left.19,5 \mathrm{~kg} / \mathrm{m}^{2}\right)^{17}$, praticantes de atividades físicas nos últimos três meses antes das coletas e portadores de distúrbios neurológicos, articulares ou musculoesqueléticos que pudessem dificultar a execução dos exercícios, ou déficit cognitivo grave, que inviabilizasse o entendimento dos procedimentos da pesquisa.

As voluntárias foram divididas aleatoriamente em dois grupos: no grupo experimental que realizou o método Pilates (grupo GP) e no grupo controle, que foi submetido a uma técnica tradicional de fortalecimento do abdome e de alongamentos estáticos (grupo GC).

A amostra foi calculada através do Programa Epi-Info 6.04, considerando uma proporção de resposta ao tratamento, no grupo GP, de $80 \%$, e no grupo GC, de $10 \%$, conforme estudo desenvolvido ${ }^{18}$, com um nível de significância de $5 \%$ e poder do teste de $80 \%$, obtendo-se sete indivíduos para cada grupo.

Para a distribuição das voluntárias nos grupos, foi utilizada a técnica de randomização em blocos de sete indivíduos, para garantir que os dois grupos apresentassem o mesmo número de participantes ${ }^{19}$. Inicialmente, foi gerada uma tabela de números aleatórios utilizando o programa Epi-Info 6.0 por um estatístico não envolvido no trabalho.

A coleta de dados foi realizada através de um formulário de registro de informações pessoais e história da prática de exercícios físicos. Em seguida, as participantes foram submetidas a um exame ultrassonográfico (para determinação da espessura dos músculos: oblíquo interno e externo, transverso do abdome e reto do abdome), uma avaliação da amplitude de movimento da coluna vertebral em flexão, extensão, rotação e flexão lateral através de testes com o flexímetro.

A avaliação ultrassonográfica foi realizada através do aparelho HD7, da marca Phillips, com transdutores convexos (C5-2), por um avaliador devidamente treinado.

A mensuração da espessura dos músculos abdominais foi feita através da distância em milímetros das fáscias superficial e profunda dos músculos transverso abdominal, oblíquo interno, oblíquo externo e reto do abdome, do lado esquerdo da voluntária, repetindo-se três vezes para ser feita a média e sempre ao final da expiração para ser controlada a influência da respiração. A mensuração foi realizada com a participante em decúbito dorsal, com os membros inferiores estendidos e os braços ao longo do corpo.

As imagens dos músculos oblíquo externo, oblíquo interno e transverso do abdome foram obtidas com o transdutor posicionado 
verticalmente à voluntária, dez centímetros à esquerda da cicatriz umbilical, no ponto médio entre a crista ilíaca e a última costela ${ }^{20}$.

A mensuração da espessura do músculo reto do abdome foi realizada posicionando o transdutor horizontalmente à voluntária, imediatamente abaixo da cicatriz umbilical ${ }^{21}$.

A avaliação da amplitude de movimento ativa da flexão, extensão, rotação e inclinação lateral da coluna torácica e lombar foi realizada através do aparelho flexímetro da marca Sanny ${ }^{\circledR}$. Para cada movimento, foram realizadas três medidas e feita a média destas.

As condutas para os grupos GP e GC foram realizadas sob orientação e supervisão contínua do pesquisador, duas vezes por semana, em um período de cinco semanas, de forma que todas as voluntárias completassem um total de dez sessões.

Cada sessão teve duração total de 50 minutos, sendo dividida em três partes: Na primeira, com duração de dez minutos, foi realizado um aquecimento com esclarecimento do método e incorporação dos princípios do Pilates, além de serem feitas mobilizações articulares globais (circundução da cervical, ombros, quadris e tornozelos) e alongamentos curtos de cadeia posterior, anterior e lateral de tronco, quadríceps e adutores de membros inferiores. Em seguida, foi iniciada a série de exercícios propriamente ditos, de aproximadamente 30 minutos (cada exercício com duração de cerca de um minuto e descanso entre eles de 30s), que foram divididos em cinco blocos.

O primeiro bloco de exercícios foi composto por: Spine Twist (rotação de tronco), Saw (serrote), The Spine Stretch (alongamento de tronco), The Spine Stretch (arredondando a coluna), The Half Roll Down (a Curva do C) com três repetições e o último exercício com seis repetições. O segundo bloco englobou o The Hundred (Cem), de início, realizou-se 20 movimentos e foi aumentando gradualmente até 100; The Shoulder Bridge (a ponte) com seis repetições; o Criss-Cross (oblíquos): com cinco repetições para cada lado.

O terceiro bloco em decúbito lateral direito composto pelos exercícios: Up and Down (sobe e desce); The Side Kick (chutes laterais): Posicionar-se na mesma forma anterior. Inspirar flexionando o quadril esquerdo e expirar voltando para a posição inicial; The Side Bend (flexão lateral) sendo os dois primeiros com cinco repetições e o último com quatro. O quarto Bloco trouxe o The One Leg Stretch (alongamento de uma perna) e One Straight Leg Stretch (alongamento da perna reta) ambos com cinco repetições em cada perna. E o quinto bloco repetiu os mesmos exercícios do terceiro bloco, porém em decúbito lateral esquerdo. E foi finalizado com dez minutos de relaxamento global com massagem.

As sessões do grupo controle tiveram como intervenção alongamentos, mantidos por 30 segundos $^{22}$, e exercícios de fortalecimento abdominal, cada um com três séries de dez repetições, sem sustentação, e com intervalos de três minutos entre as séries.

Primeiramente foram realizados os alongamentos de cadeia lateral do tronco, em ortostatismo. Em seguida foi alongada a cadeia anterior do tronco, em decúbito ventral, continuando com o alongamento dos rotadores da coluna, em decúbito dorsal, e finalizando com a cadeia posterior, na posição sentada.

O fortalecimento foi iniciado com abdominais tradicionais, com os dois pés no solo e as mãos atrás das orelhas e os ombros abduzidos, flexionando o tronco até desencostar o ângulo inferior da escápula do solo. Em seguida, no mesmo posicionamento anterior, foram feitos os abdominais oblíquos, levando um ombro em direção ao joelho contralateral. Por fim, foram realizados os abdominais para a porção inferior do músculo reto do abdome, com a cabeça e os membros superiores apoiados no solo e os MMII em $90^{\circ}$ de flexão de quadril e joelho, com os pés suspensos, deixando o quadril cerca de $30^{\circ} \mathrm{em}$ direção ao solo, e depois flexionando em direção ao tórax ${ }^{23}$. Ao final, também passaram por dez minutos de relaxamento global com massagem.

\section{RESULTADOS}

Das 29 mulheres recrutadas para esta pesquisa, 14 se enquadraram nos critérios de elegibilidade, no entanto, após o início das intervenções houve uma perda, conforme fluxograma (figura 1 e tabelas 1-4), resultando numa amostra final de 13 participantes, obtendo-se seis no GP e sete no GC.

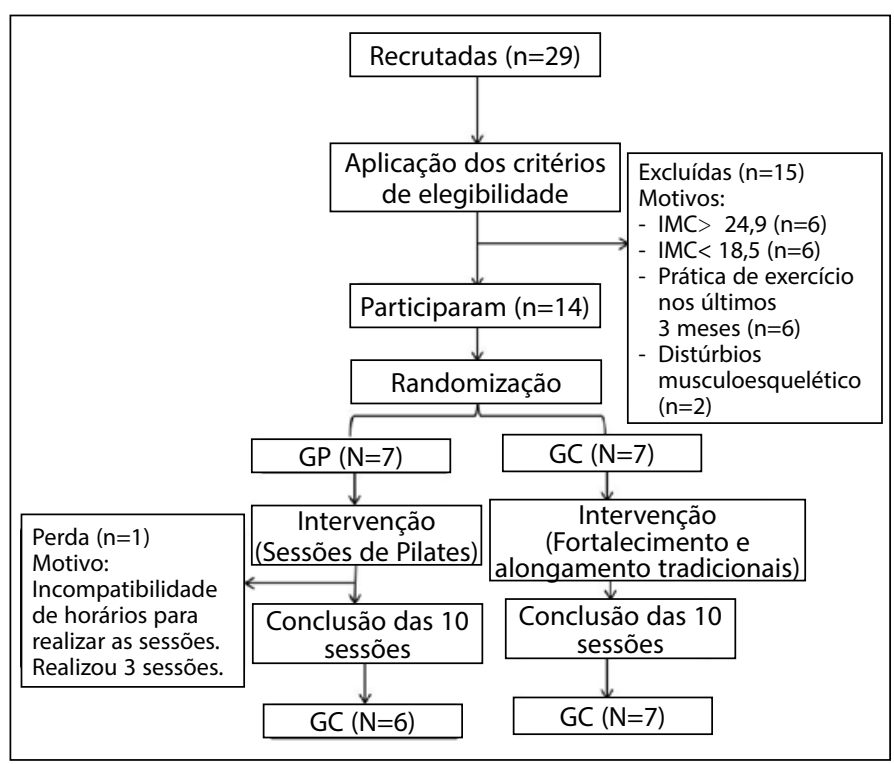

Figura 1. Fluxograma da pesquisa.

Tabela 1. Comparação dos dados referentes à caracterização da amostra entre os grupos.

\begin{tabular}{|c|c|c|c|}
\hline Caracterização da amostra & GP & GC & Valor de $\mathrm{P}$ \\
\hline Idade (Média_Desvio-Padrão) & $22 \pm 0,89$ & $21,3 \pm 2,25$ & $0,882^{\mathrm{a}}$ \\
\hline Peso (Kg) (Média_Desvio-Padrão) & $58,9 \pm 6,29$ & $53,8 \pm 4,28$ & $0,116^{\mathrm{a}}$ \\
\hline Altura (m) (Média_Desvio-Padrão) & $1,63 \pm 0,63$ & $1,60 \pm 0,03$ & $0,548^{b}$ \\
\hline IMC (Kg/m2) (Média_Desvio-Padrão) & $22,0 \pm 2,57$ & $20,9 \pm 1,74$ & $0,274^{b}$ \\
\hline
\end{tabular}

teste t-student; ${ }^{\mathrm{b}}$ Teste de Mann-Whitney.

Tabela 2. Comparação das Medidas Ultrassonográficas e de Flexibilidade entre os grupos antes da intervenção.

\begin{tabular}{c|c|c|c}
\hline $\begin{array}{c}\text { Medidas ultrassonográficas } \\
\text { e de flexibilidade } \\
\text { (Média+Desvio-padrão) }\end{array}$ & \multicolumn{2}{|c|}{ Antes das intervenções } & \multirow{2}{*}{$\begin{array}{c}\text { Valor de P } \\
\text { (entre os grupos) }\end{array}$} \\
\cline { 2 - 3 } & GP & GC & $0,776^{\mathrm{a}}$ \\
\hline Transverso abdominal (mm) & $0,34 \pm 0,03$ & $0,33 \pm 0,07$ & $0,712^{\mathrm{a}}$ \\
\hline Oblíquo interno (mm) & $0,57 \pm 0,05$ & $0,61 \pm 0,13$ & $0,821^{\mathrm{b}}$ \\
\hline Oblíquo externo (mm) & $0,48 \pm 0,05$ & $0,47 \pm 0,04$ & $0,559^{\mathrm{b}}$ \\
\hline Reto abdominal (mm) & $0,84 \pm 0,08$ & $0,93 \pm 0,18$ & $0,128^{\mathrm{a}}$ \\
\hline Rotação direita (graus) & $53,83 \pm 14,72$ & $68,44 \pm 21,01$ & $0,279^{\mathrm{a}}$ \\
\hline Rotação esquerda (graus) & $56,44 \pm 13,85$ & $64,27 \pm 17,95$ & $0,253^{\mathrm{b}}$ \\
\hline Flexão anterior (graus) & $62,16 \pm 14,19$ & $71,33 \pm 12,86$ & $0,65^{\mathrm{a}}$ \\
\hline Extensão (graus) & $39,16 \pm 9,15$ & $42,88 \pm 12,42$ & $0,994^{\mathrm{a}}$ \\
\hline Inclinação direita (graus) & $44,39 \pm 9,55$ & $44,67 \pm 13,01$ & $0,861^{\mathrm{a}}$ \\
\hline Inclinação esquerda (graus) & $42,83 \pm 9,33$ & $43,83 \pm 10,21$ & \\
\hline
\end{tabular}

${ }^{a}$ teste t-student; ${ }^{b}$ Teste de Mann-Whitney. 
Tabela 3. Comparação das medidas ultrassonográficas e de flexibilidade entre os grupos após a intervenção.

\begin{tabular}{c|c|c|c}
\hline \multirow{2}{*}{$\begin{array}{c}\text { Medidas ultrassonográficas } \\
\text { e de flexibilidade } \\
\text { (Média+Desvio-padrão) }\end{array}$} & \multicolumn{2}{|c|}{ Após as intervenções } & \multirow{2}{*}{$\begin{array}{c}\text { Valor de P } \\
\text { (entre os grupos) }\end{array}$} \\
\cline { 2 - 3 } & GP & GC & $0,601^{\text {a }}$ \\
\hline Transverso abdominal (mm) & $0,35 \pm 0,04$ & $0,35 \pm 0,10$ & $0,943^{b}$ \\
\hline Oblíquo interno (mm) & $0,60 \pm 0,09$ & $0,64 \pm 0,12$ & $0,785^{a}$ \\
\hline Oblíquo externo (mm) & $0,55 \pm 0,05$ & $0,52 \pm 0,06$ & $0,476^{a}$ \\
\hline Reto abdominal (mm) & $0,95 \pm 0,09$ & $0,91 \pm 0,16$ & $0,206^{\mathrm{a}}$ \\
\hline Rotação direita (graus) & $65,83 \pm 14,97$ & $73,78 \pm 13,25$ & $0,097^{\mathrm{a}}$ \\
\hline Rotação esquerda (graus) & $65,44 \pm 14,20$ & $80,44 \pm 19,25$ & $0,167^{\mathrm{a}}$ \\
\hline Flexão anterior (graus) & $69,72 \pm 18,80$ & $80,72 \pm 6,32$ & $0,561^{\mathrm{a}}$ \\
\hline Extensão (graus) & $49,27 \pm 8,59$ & $46,77 \pm 4,31$ & $0,503^{\mathrm{a}}$ \\
\hline Inclinação direita (graus) & $48,72 \pm 10,72$ & $51,11 \pm 10,11$ & $0,972^{\mathrm{a}}$ \\
\hline Inclinação esquerda (graus) & $48,28 \pm 7,44$ & $47,06 \pm 5,93$ &
\end{tabular}

Tabela 4. Comparação intragrupo das medidas ultrassonográficas e de flexibilidade antes e após a intervenção.

\begin{tabular}{c|c|c}
\hline \multirow{2}{*}{$\begin{array}{c}\text { Medidas ultrassonográficas } \\
\text { e de flexibilidade }\end{array}$} & \multicolumn{2}{|c}{$\begin{array}{c}\text { Comparação intragrupo } \\
\text { (antes e após a intervenção) }\end{array}$} \\
\cline { 2 - 3 } & GP (Valor de P) & GC (Valor de P) \\
\hline Transverso abdominal & $0,522^{\mathrm{a}}$ & $0,518^{\mathrm{a}}$ \\
\hline Oblíquo interno & $0,413^{\mathrm{a}}$ & $0,343^{\mathrm{b}}$ \\
\hline Oblíquo externo & $0,413^{\mathrm{a}}$ & $0,098^{\mathrm{b}}$ \\
\hline Reto abdominal & $0,017^{\mathrm{b}}$ & $0,807^{\mathrm{a}}$ \\
\hline Rotação direita & $0,002^{\mathrm{a}}$ & $0,449^{\mathrm{a}}$ \\
\hline Rotação esquerda & $0,001^{\mathrm{a}}$ & $0,044^{\mathrm{a}}$ \\
\hline Flexão anterior & $0,542^{\mathrm{a}}$ & $0,173^{\mathrm{b}}$ \\
\hline Extensão & $0,066^{\mathrm{a}}$ & $0,46^{\mathrm{a}}$ \\
\hline Inclinação direita & $0,329^{\mathrm{a}}$ & $0,073^{\mathrm{a}}$ \\
\hline Inclinação esquerda & $0,037^{\mathrm{a}}$ & $0,254^{\mathrm{a}}$ \\
\hline
\end{tabular}

Teste T pareado; ${ }^{b}$ Teste de Wilcoxon.

\section{DISCUSSÃO}

Em relação à ausência de respostas dos músculos transverso do abdome e oblíquos interno e externo aos exercícios do Pilates, uma revisão sistemática ${ }^{24}$ realizada com pessoas saudáveis mostra que não há evidências suficientes de que o método incremente a espessura da musculatura abdominal. Outros autores ${ }^{25-27}$ sugerem que os exercícios de Pilates tem como foco trabalhar a estabilidade da coluna e recuperar o comando motor proprioceptivo dos músculos, em especial o transverso do abdome, principal estabilizador abdominal da coluna, assim como o oblíquo interno e oblíquo externo, que atuam como auxiliares nesta função ${ }^{28}$. Dessa forma, através dos exercícios de Pilates, não se enfoca o aumento de trofismo desses músculos além das dimensões consideradas normais para sexo, idade e altura do indivíduo, mas sim, a melhora do comando motor.

Entretanto, resultados de aumento significativo de espessura dos músculos posturais só podem ser observados em indivíduos que apresentem previamente alguma redução do trofismo, assim como ocorre em indivíduos com lombalgia e hérnia de disco, onde é observada uma diminuição da espessura da musculatura estabilizadora da coluna29,30.

Assim, tanto o presente estudo quanto a maioria das pesquisas envolvendo o Pilates, foram desenvolvidos com indivíduos saudáveis, que não apresentam uma redução significativa desse trofismo antes da intervenção.

A constatação dessa diferença em relação à espessura muscular pode ser melhor evidenciada em estado de contração ${ }^{31}$. Em pesquisa com mulheres de 23 a 37 anos, observaram que, após 16 sessões, houve o aumento do tamanho dos músculos transverso abdominal e oblíquo interno observado pela ultrassonografia somente durante a contração voluntária do músculo, no momento da execução dos exercícios de Pilates. Na avaliação em repouso, os mesmos autores não encontraram diferenças após a intervenção, conforme foi verificado no presente estudo, que realizou dez sessões. Isso se deve ao fato que, durante o exercício, houve uma melhor ativação do músculo após a finalização das sessões de Pilates, resultando num melhor comando motor e, consequentemente, maior recrutamento do músculo durante a contração.

Já com relação ao reto abdominal, o presente estudo observou um efeito de hipertrofia, mesmo em repouso, no grupo Pilates. Essa resposta se deve ao fato que este músculo tem a função de mobilidade, apresentando mudanças mais significativas frente à carga imposta. Estudo que avaliou os músculos abdominais por ressonância magnética antes e após 72 sessões de Pilates em mulheres saudáveis e sedentárias. Demonstrou um aumento na espessura de todos esses músculos, entretanto, houve um percentual de ganho significativo no músculo reto abdominal (21\%)'

Em relação aos resultados de flexibilidade do grupo Pilates, a maioria dos exercícios utilizados para este grupo, nesta pesquisa, envolveu manutenção de postura com estabilização da coluna, associados a movimento de tronco e MMII. Para se ter ganhos de flexibilidade, outros pesquisadores ${ }^{22}$ sugerem que o músculo a ser alongado deve ser colocado uma situação de estiramento máximo e mantê-lo por, pelo menos, 30 segundos. O aumento observado nas médias das rotações e da inclinação, pode ter se dado ao fato que, após as sessões de Pilates, houve um aumento da segurança na realização deste movimento em decorrência da estabilidade obtida pela coluna, permitindo que haja uma maior amplitude nos pequenos movimentos intervertebrais durante a realização do teste.

Em relação ao uso do ultrassom para avaliação do trofismo abdominal, há possibilidade de que mudanças ocorridas na estrutura ou na forma dos músculos não tenham sido visualizadas através deste aparelho. Talvez, o uso da Ressonância Magnética (RM), por exemplo, poderia ter detectado um maior ganho ou mudança muscular, visto que, a RM, no diagnóstico por imagem, é a que tem a melhor definição e contraste entre as estruturas de partes moles. Neste contexto, em uma pesquisa com mulheres saudáveis submetidas á sessões de Pilates, conseguiu detectar aumento significativo do trofismo abdominal após as intervenções através da RM, o que corrobora com este pensamento'.

Outro fator que pode ter influenciado na semelhança de resposta do GP e GC após as intervenções em relação ao trofismo muscular foi o hemicorpo avaliado. Pois, o lado dominante parece ter maiores diferenças de ganho de espessura da musculatura abdominal ipsilateral, em comparação com o lado não-dominante 1 . Devido à todas as medidas do presente estudo terem sido feitas do lado esquerdo, e todas as voluntárias serem destras, este fator pode ter sido fundamental nos resultados encontrados nesta pesquisa, podendo ter ocorrido uma subestimação dos valores reais de ganho de trofismo da musculatura abdominal das voluntárias.

Em relação ao tempo de repouso entre os exercícios, Hides et al. e 
Critchley et al. ${ }^{30,31}$ recomendam de dois a três minutos para programas visando à hipertrofia muscular, sugerem que as pausas deveriam permanecer entre um e três minutos ${ }^{33,34}$. Seguindo estas recomendações, o grupo controle do presente estudo obteve três minutos de repouso entre as séries, enquanto no grupo Pilates, as participantes descansavam 30 segundos entre um exercício e outro. E isso pode ter contribuído para a fadiga no GP e ter dificultado sua resposta de hipertrofia.

\section{CONCLUSÃO}

Algumas limitações do estudo devem ser consideradas, como a mensuração do trofismo abdominal ter sido realizada apenas de um lado. Devido à quantidade de medidas a serem avaliadas, não foi viável incluir os dois lados. Há também a questão da mensuração ter sido realizada apenas no repouso.
Era esperado que houvesse mudanças estruturais no músculo, portanto, não seria necessário avaliar o músculo em contração. Por fim, o número de sessões pode ter sido insuficiente para que ocorressem mudanças no trofismo abdominal e na flexibilidade do tronco. Sugere-se que, em estudos futuros, estas questões sejam levadas em consideração, a fim de que resultados mais significativos sejam encontrados.

Desta forma, destaca-se a importância de investir em pesquisas envolvendo o Pilates, principalmente com a utilização de métodos de imagem mais acurados, e com um maior tempo de intervenção, para que se possa avaliar o efeito do método.

Todos os autores declararam não haver qualquer potencial conflito de interesses referente a este artigo.

\section{REFERÊNCIAS}

1. Dorado C, Calbet JA, Lopez-Gordillo A, Alayon S, Sanchis-Moysi, J. Marked effects of Pilates on the abdominal muscles: a longitudinal magnetic resonance imaging study. Medicine and science in sports and exercise. Med Sci Sports Exerc. 2012;44(8):1589-1594.

2. Conceição JS, Mergener CR. Eficácia do método Pilates no solo em pacientes com lombalgia crônica. Relato de casos. Rev Dor. 2012;13(4):385-8.

3. Bertolla F, Baroni BM, Junior ECPL, Oltramari JD. Efeito de um programa de treinamento utilizando o método Pilates na flexibilidade de atletas juvenis de futsal. Rev Bras Med Esporte. 2007;13(4):222-6.

4. Pinheiro KR, Rocha TC, Brito NM, Silva MLG, Carvalho M, Mesquita LS, et al. Influence of pilates exercises on soil stabilization in lumbar muscles in older adults. Rev Bras Cineantropom Desempenho Hum. 2014; 16(6):648-57.

5. Silva AC, Mannrich G. Pilates na Reabilitação: uma revisão sistemática. Fisioter Mov. 2009;23(9):499-55.

6. Miranda LB. Morais PDC. Efeito do método pilates sobre a composição corporal e flexibilidade. RBPFEX. 2009;3(13):16-21

7. Sinzato CR, Taciro C, Pio CA, Toledo AM, Cardoso JR, Carregaro RL. Efeitos de 20 sessões do método Pilates no alinhamento postural e flexibilidade de mulheres jovens: estudo piloto. Fisioter Pesqui. 2013;20(2):143-50

8. Souza EF, Cantergi D, Mendonça A, Kennedy C, Loss JF. Análise eletromiográfica dos músculos reto femoral e reto abdominal durante a execução dos exercícios hundred e teaser do método pilates. Rev Bras Med Esporte. 2012;18(2):105-8.

9. Sacco IC, Andrade MS, Souza PS, Nisiyama M, Cantuária AL, Maeda FY, et al. Método Pilates em revista: aspectos biomecânicos de movimentos específicos para reestruturação postural-estudos de caso. Rev Bras Ci e Mov. 2005;13:65-78.

10. Lynch JA, Chalmers GR, Knutzen KM, Martin LT. Effect on performance of learning a pilates skill with or without a mirror. J Bodyw Mov Ther. 2009;13(3):283-90.

11. Abe T, DeHoyos DV, Pollock ML, Garzarella L. Time course for strength and muscle thickness changes following upper and lower body resistance training in men and women. Eur J Appl Physiol. 2000;81(3):174-80

12. Gurney JM, Jelliffe DB. Arm anthropometry in nutritional assessment. Nomogram for rapid calculation of muscle circumference and cross-sectional muscle and fat áreas. Am J Clin Nutr. 1973;26(9):912-5.

13. Heymsfield SB, McManus C, Smith J, Stevens V, Nixon DW. Anthropometric measurement of muscle mass: revised equations for calculating bone-free arm muscle área. Am. J. Clin. Nutr. 1982;36(4):680-90.

14. Maughan $\mathrm{Rj}$, et al. Relationships between muscle strength and muscle cross-sectional área in male sprinters and endurance runners. Eur. J. Appl. Physiol. 1983;50(3):309-18.

15. Siqueira GR, Alencar GG, Oliveira NK, Leite FN. A eficácia da estabilização segmentar vertebral no aumento do trofismo dos multífidos e melhora da dor em portadores de hérnia discal lombar. Rev Bras Ci e Mov. 2014;22(1):81-9.

16. Junior AA, Nascimento MA, Franco R, Silva VP, Martins VF, Guariglia DA. Comparação e concordância de instrumentos de avaliação da amplitude de movimento da coluna cervical de homens universitários. Rev Educ Fis. 2013;24(4):609-16.

17. Organização Mundial de Saúde. Programa Peso Saudável. [Atualizado em 24 de Maio de 2013].
Disponivel em: http://www.paho.org/bra/index.php?option=com content\&view=article\&id=3213\&l temid=0. Acesso em: 20 nov 2014

18. Araújo A, Silva MEB, Mello EB, Cader D, Salgado SASI, Dantas A, et al. The effectiveness of the Pilates method: reducing the degree of non-structural scoliosis, and improving flexibility and pain in female college students. J Bodyw Mov Ther. 2012;16(2):191-8.

19. Escosteguy CC. Tópicos Metodológicos e Estatísticos em Ensaios Clínicos Controlados Randomizados. Arq Bras Cardiol. 1999;72(2):139-143.

20. Koppenhaver SL, Hebert JJ, Fritz JM, Parent EC, Teyhen DS, Magel JS. Reliability of rehabilitative ultrasound imaging of the transversus abdominis and lumbar multifidus muscles. Arch Phys Med Rehabil. 2009;90(1):87-94

21. Connell D, Ali K, Javid M, Bell P, Batt M, Kemp S. Sonography and MRI of rectus abdominis muscle strain in elite tennis players. AJR Am J Roentgenol. 2006;187(6):1457-61.

22. Bandy WD, Irion JM, Briggler M. The effect of time and frequency of static stretching on flexibility of the hamstring muscles. Phys Ther. 1997;77(10):1090-6.

23. Pardal DM, Sacco IC, Serrão JC, Amadio AC. Comparação de Atividade Eletromiográfica de músculos abdominais durante exercícios convencionais. BJB. 2003;4(6):29-37.

24. Cruz-Ferreira A, Fernandes J, Laranjo L, Bernardo LM, Silva A. A systematic review of the effects of pilates method of exercise in healthy people. Archives of physical medicine and rehabilitation. Arch Phys Med Rehabil. 2011;92(12):2071-81.

25. Loss JF, Melo MO, Rosa CH, Santos AB, Torre ML, Silva YO. Atividade elétrica dos músculos oblíquos externos e multífidos durante o exercício de flexoextensão do quadril realizado no Cadillac com diferentes regulagens de mola e posições do indivíduo. Rev Bras Fisioter. 2010;14(6):510-7.

26. Marés G, Oliveira KB, Piazza MC, Preis C, Neto LB. A importância da estabilização central no método Pilates: uma revisão sistemática. Fisioter Mov. 2012;25(2):445-51.

27. Cruz-Ferreira A, Fernandes J, Kuo YL, Bernardo LM, Fernandes O, Laranjo L, Silva A. Does pilates-based exercise improve postural alignment in adult women? Women Health. 2013;53(6):597-611.

28. Panjabi MM. Clinical spinal instability and low back pain. J Electromyogr Kinesiol. 2003;13(4):371-9.

29. Leinonen V, Kankaanpaa M, Luukkonen M, Kansanen M, Hanninen O, Airaksinen O, et al. Lumbar paraspinal muscle function, perception of lumbar position, and postural control in disc herniationrelated back pain. Spine. 2003;28(8):842-8.

30. Hides JA, Stokes MJ, Saide M, Jull GA, Cooper DH. Evidence of lumbar multifidus muscle wasting ipsilateral to symptoms in patients with acute/subacute low back pain. Spine. 1994;19(2):165-72.

31. Critchley DJ, Pierson Z, Battersby G. Effect of pilates mat exercises and conventional exercise programmes on transversus abdominis and obliquus internus abdominis activity: pilot randomised trial. Man Ther. 2011;16(2):183-9.

32. Güllich A, Schmidtbleicher D. Struktur der Kraftfähigkeiten und ihrer Trainings- methoden. Dtsch Z Sportmed.1999;50(8):223-34.

33. American College of Sports Medicine-ACSM. Position stand on progression models in resistance training for healthy adults. Exercise and physical activity for older adults. Med Sci Sports Exerc. 2002;34(2):364-80

34. Kraemer WJ, Ratamess NA. Fundamentals of resistance training: progression and exercise prescription. Med Sci Sports Exerc. 2004;36(4):674-88 